\title{
A NUMERICAL SOLUTION OF A FRACTIONAL OSCILLATOR EQUATION WITH VARIOUS TYPES OF BOUNDARY CONDITIONS
}

\author{
Tomasz Błaszczyk ${ }^{1}$, Jaroslaw Siedlecki ${ }^{1}$, Mariusz Ciesielski ${ }^{2}$ \\ ${ }^{I}$ Institute of Mathematics, Czestochowa University of Technology \\ Czestochowa, Poland \\ ${ }^{2}$ Institute of Computer and Information Sciences, Czestochowa University of Technology \\ Czestochowa, Poland \\ tomasz.blaszczyk@im.pcz.pl,jaroslaw.siedlecki@im.pcz.pl,mariusz.ciesielski@icis.pcz.pl
}

\begin{abstract}
In this paper we study the fractional differential equation, containing both the left and right fractional derivatives, with respect to various types of boundary conditions. We transform the fractional differential equation into equivalent integral form. Next, we develop a discrete form of the composition of the left and right fractional integrals, based on the trapezoidal rule of integration, and we obtain a numerical scheme for a fractional integral equation. The discrete form of integral equation is rewritten in the matrix form. Finally, several examples of computations are presented.
\end{abstract}

Keywords: numerical method, fractional operators, oscillator equation, boundary value problem

\section{Introduction}

Fractional differential equations, containing both the left and right fractional derivatives (Caputo or/and Riemann-Liouville), have become more and more interesting over the last years for scientists. They are well studied from the theoretical point of view (we refer the reader to books [1-3] and articles [4-9]). Furthermore, one can find several papers devoted to applications of these types of equations in various fields of science: anomalous diffusion $[8,10,11]$, fractional continuum mechanics $[12,13]$, fractional oscillators $[14,15]$ etc.

One of the most important problems is how to find a solution of the fractional differential equations including simultaneously the left and right operators. The problem is quite comprehensive due to the fact that the equations can be analyzed with different types of operators (Caputo or/and Riemann-Liouville) and various types of boundary conditions, like Dirichlet, Neumann, Robin, natural, fractional or mixed conditions.

For some particular cases, an exact solution of the above-mentioned problems is known [1,5]. However, in general, an analytical solution is not available. Even if it is known, the form of solution does not allow for its effective use. For this reason, numerical methods devoted to the Fractional Euler-Lagrange Equations (FELE) are becoming more important and attractive for scientists [10, 15-20]. 


\section{Preliminaries}

In this section we recall basic definitions and some properties of fractional operators [21-23]. We start from definitions of the left- and right-sided Riemann-Liouville fractional derivative operators for $\alpha>0$

$$
\begin{gathered}
D_{a^{+}}^{\alpha} y(x):= \begin{cases}D^{m} I_{a^{+}}^{m-\alpha} y(x) & \text { for } m-1<\alpha<m \\
D^{m} y(x) & \text { for } \alpha=m\end{cases} \\
D_{b^{-}}^{\alpha} y(x):= \begin{cases}(-1)^{m} D^{m} I_{b^{-}}^{m-\alpha} y(x) & \text { for } m-1<\alpha<m \\
(-1)^{m} D^{m} y(x) & \text { for } \alpha=m\end{cases}
\end{gathered}
$$

The left- and right-sided Caputo fractional derivatives are defined as follows

$$
\begin{gathered}
{ }^{C} D_{a^{+}}^{\alpha} y(x):= \begin{cases}I_{a^{+}}^{m-\alpha} D^{m} y(x) & \text { for } m-1<\alpha<m \\
D^{m} y(x) & \text { for } \alpha=m\end{cases} \\
{ }^{C} D_{b^{-}}^{\alpha} y(x):= \begin{cases}(-1)^{m} I_{b^{-}}^{m-\alpha} D^{m} y(x) & \text { for } m-1<\alpha<m \\
(-1)^{m} D^{m} y(x) & \text { for } \alpha=m\end{cases}
\end{gathered}
$$

where $D^{m}$ denotes the $m$-th order derivative and operators $I_{a^{+}}^{\alpha}$ and $I_{b^{-}}^{\alpha}$ are respectively the left- and right-sided fractional integrals of order $\alpha>0$ defined by

$$
\begin{array}{ll}
I_{a^{+}}^{\alpha} y(x):=\frac{1}{\Gamma(\alpha)} \int_{a}^{x} \frac{y(\tau)}{(x-\tau)^{1-\alpha}} d \tau \text { for } x>a \\
I_{b^{-}}^{\alpha} y(x):=\frac{1}{\Gamma(\alpha)} \int_{x}^{b} \frac{y(\tau)}{(\tau-x)^{1-\alpha}} d \tau & \text { for } x<b
\end{array}
$$

The following relationships between both operators (Caputo and Riemann-Liouville derivatives) occur [21-23]

$$
{ }^{C} D_{a^{+}}^{\alpha} y(x)=D_{a^{+}}^{\alpha} y(x)-\sum_{k=0}^{m-1} \frac{y^{(k)}(a)}{\Gamma(k-\alpha+1)}(x-a)^{k-\alpha}
$$




$$
{ }^{C} D_{b^{-}}^{\alpha} y(x)=D_{b^{-}}^{\alpha} y(x)-\sum_{k=0}^{m-1} \frac{y^{(k)}(b)}{\Gamma(k-\alpha+1)}(b-x)^{k-\alpha}
$$

Composition rules of fractional operators are as follows [21-23]

$$
\begin{gathered}
I_{a^{+}}^{\alpha C} D_{a^{+}}^{\alpha} y(x)=y(x)-\sum_{k=0}^{m-1} \frac{y^{(k)}(a)}{k !}(x-a)^{k} \\
I_{b^{-}}^{\alpha C} D_{b^{-}}^{\alpha} y(x)=y(x)-\sum_{k=0}^{m-1} \frac{(-1)^{k} y^{(k)}(b)}{k !}(b-x)^{k} \\
D_{a^{+}}^{\beta} I_{a^{+}}^{\alpha} y(x)= \begin{cases}I_{a^{+}}^{\alpha-\beta} y(x) & \text { for } \alpha>\beta \\
y(x) & \text { for } \alpha=\beta \\
D_{a^{+}}^{\beta-\alpha} y(x) & \text { for } \alpha<\beta\end{cases}
\end{gathered}
$$

The fractional integration of a constant gives us

$$
I_{a^{+}}^{\alpha} C=C \frac{(x-a)^{\alpha}}{\Gamma(1+\alpha)}
$$

and fractional differentiation of a power function leads to

$$
D_{a^{+}}^{\beta}(x-a)^{\alpha}=\frac{\Gamma(1+\alpha)}{\Gamma(1+\alpha-\beta)}(x-a)^{\alpha-\beta}
$$

\section{Main problem}

We study the following FELE

$$
{ }^{C} D_{b^{-}}^{\alpha} D_{a^{+}}^{\alpha} y(x)-\omega^{2 \alpha} y(x)=0
$$

with boundary conditions given in the following forms

$$
y(a)=0,\left.\quad D_{a^{+}}^{\beta} y(x)\right|_{x=b}=L_{\beta}
$$

where $1 / 2<\alpha \leq 1,0 \leq \beta \leq 1$ and $\omega \geq 0$. For $\beta=0$, the right-side boundary condition is treated as the Dirichlet type $\left.D_{a^{+}}^{0} y(x)\right|_{x=b}=y(b)=L_{0}$ and for $\beta=1$, this condition is the Neumann type $\left.D_{a^{+}}^{1} y(x)\right|_{x=b}=y^{\prime}(b)=L_{1}$. 
In the particular case for $\alpha=1$, the composition of differential operators is reduced to ${ }^{C} D_{b^{-}}^{1} D_{a^{+}}^{1} y(x)=-y^{\prime \prime}(x)$ and then Eq. (14) can be written as

$$
y^{\prime \prime}(x)+\omega^{2} y(x)=0
$$

One can find the solution of Eq. (16) related to boundary conditions (15) using an analytical method. We derived the solution in the form

$$
y(x)= \begin{cases}L_{\beta} \frac{\sin (\omega(x-a))}{\left.D_{a^{+}}^{\beta} \sin (\omega(x-a))\right|_{x=b}} & \text { for } \omega>0 \\ L_{\beta} \frac{x-a}{\left.D_{a^{+}}^{\beta}(x-a)\right|_{x=b}}=L_{\beta} \frac{\Gamma(2-\beta)}{(b-a)^{1-\beta}}(x-a) & \text { for } \omega=0\end{cases}
$$

where

$$
\left.D_{a^{+}}^{\beta} \sin (\omega(x-a))\right|_{x=b}= \begin{cases}\sin (\omega(b-a)) & \text { for } \beta=0 \\ \sum_{n=0}^{\infty} \frac{(-1)^{n} \omega^{2 n+1}}{\Gamma(2 n+2-\beta)}(b-a)^{2 n+1-\beta} & \text { for } 0<\beta<1 \\ \omega \cos (\omega(b-a)) & \text { for } \beta=1\end{cases}
$$

One can note that for the integer values of parameter $\beta$, this analytical solution has the forms known in literature.

In the other cases, the solutions of the boundary value problem (14)-(15) we will find in a numerical way.

\section{Transformation of the BVP (14)-(15) to the integral form}

To solve the analysed problem (14)-(15), we will transform the fractional differential equation into adequate integral equation.

By using the fractional integral operator $I_{a^{+}}^{\alpha} I_{b^{-}}^{\alpha}$ we can write an equivalent form of Eq. (14)

$$
I_{a^{+}}^{\alpha} I_{b^{-}}^{\alpha C} D_{b^{-}}^{\alpha} D_{a^{+}}^{\alpha} y(x)-\omega^{2 \alpha} I_{a^{+}}^{\alpha} I_{b^{-}}^{\alpha} y(x)=0
$$

Next we use the composition rule of fractional operators (10)

$$
I_{a^{+}}^{\alpha}\left[D_{a^{+}}^{\alpha} y(x)-\left.D_{a^{+}}^{\alpha} y(x)\right|_{x=b}\right]-\omega^{2 \alpha} I_{a^{+}}^{\alpha} I_{b^{-}}^{\alpha} y(x)=0
$$


and by applying the properties (9) and (11) we get

$$
y(x)-y(a)-\left.D_{a^{+}}^{\alpha} y(x)\right|_{x=b} \frac{(x-a)^{\alpha}}{\Gamma(\alpha+1)}-\omega^{2 \alpha} I_{a^{+}}^{\alpha} I_{b^{-}}^{\alpha} y(x)=0
$$

Denoting by $C=\left.D_{a^{+}}^{\alpha} y(x)\right|_{x=b}$ and for $y(a)=0$, Eq. (21) takes the form

$$
y(x)-\omega^{2 \alpha} I_{a^{+}}^{\alpha} I_{b^{-}}^{\alpha} y(x)=C \frac{(x-a)^{\alpha}}{\Gamma(\alpha+1)}
$$

The unknown value of $C$ should be determined on the basis of the right-side boundary condition. In this order, we differentiate Eq. (22) by using the fractional operator $D_{a^{+}}^{\beta}$. Then we obtain

$$
D_{a^{+}}^{\beta} y(x)-\omega^{2 \alpha} D_{a^{+}}^{\beta} I_{a^{+}}^{\alpha} I_{b^{-}}^{\alpha} y(x)=C \frac{D_{a^{+}}^{\beta}(x-a)^{\alpha}}{\Gamma(\alpha+1)}
$$

or, after simplifications (we applied the rule (13)) we have

$$
D_{a^{+}}^{\beta} y(x)-\omega^{2 \alpha} D_{a^{+}}^{\beta} I_{a^{+}}^{\alpha} I_{b^{-}}^{\alpha} y(x)=C \frac{(x-a)^{\alpha-\beta}}{\Gamma(\alpha-\beta+1)}
$$

Next, we put the value $x=b$ into Eq. (24) and then we calculate the value $C$

$$
\begin{aligned}
C & =\frac{\Gamma(\alpha-\beta+1)}{(b-a)^{\alpha-\beta}}\left(\left.D_{a^{+}}^{\beta} y(x)\right|_{x=b}-\left.\omega^{2 \alpha} D_{a^{+}}^{\beta} I_{a^{+}}^{\alpha} I_{b^{-}}^{\alpha} y(x)\right|_{x=b}\right) \\
& =\frac{\Gamma(\alpha-\beta+1)}{(b-a)^{\alpha-\beta}}\left(L_{\beta}-\left.\omega^{2 \alpha} D_{a^{+}}^{\beta} I_{a^{+}}^{\alpha} I_{b^{-}}^{\alpha} y(x)\right|_{x=b}\right)
\end{aligned}
$$

In Eq. (25) the part containing $\left.D_{a^{+}}^{\beta} I_{a^{+}}^{\alpha} I_{b^{-}}^{\alpha} y(x)\right|_{x=b}$ occurs. Depending on the values $\alpha$ and $\beta$, the following three cases are distinguished

$$
\left.D_{a^{+}}^{\beta} I_{a^{+}}^{\alpha} I_{b^{-}}^{\alpha} y(x)\right|_{x=b}= \begin{cases}\left.I_{a^{+}}^{\alpha-\beta} I_{b^{-}}^{\alpha} y(x)\right|_{x=b} & \text { for } \alpha>\beta \\ \left.I_{a^{+}}^{0} I_{b^{-}}^{\alpha} y(x)\right|_{x=b}=\left.I_{b^{-}}^{\alpha} y(x)\right|_{x=b}=0 & \text { for } \alpha=\beta \\ \left.D_{a^{+}}^{\beta-\alpha} I_{b^{-}}^{\alpha} y(x)\right|_{x=b} & \text { for } \alpha<\beta\end{cases}
$$


Finally, we substitute the value $C$ (Eq. (25)) into Eq. (22) and we get the integral form of the considered Eq. (12)

$$
\begin{aligned}
& y(x)-\omega^{2 \alpha}\left[I_{a^{+}}^{\alpha} I_{b^{-}}^{\alpha} y(x)-\frac{\Gamma(\alpha-\beta+1)}{\Gamma(\alpha+1)} \frac{(x-a)^{\alpha}}{(b-a)^{\alpha-\beta}} \times\left\{\begin{array}{ll}
\left.I_{a^{+}}^{\alpha-\beta} I_{b^{-}}^{\alpha} y(x)\right|_{x=b} & \text { for } \alpha>\beta \\
0 & \text { for } \alpha=\beta \\
\left.D_{a^{+}}^{\beta-\alpha} I_{b^{-}}^{\alpha} y(x)\right|_{x=b} & \text { for } \alpha<\beta
\end{array}\right]\right. \\
& =\frac{\Gamma(\alpha-\beta+1)}{\Gamma(\alpha+1)} \frac{(x-a)^{\alpha}}{(b-a)^{\alpha-\beta}} L_{\beta}
\end{aligned}
$$

\section{Particular cases:}

Depending on the parameter $\beta$, Eq. (27) can be simplified to the following forms:

Case I: $\beta=0$ (this corresponds to the boundary condition $y(b)=L_{0}$ )

$$
y(x)-\omega^{2 \alpha}\left[I_{a^{+}}^{\alpha} I_{b^{-}}^{\alpha} y(x)-\left.\frac{(x-a)^{\alpha}}{(b-a)^{\alpha}} I_{a^{+}}^{\alpha} I_{b^{-}}^{\alpha} y(x)\right|_{x=b}\right]=\frac{(x-a)^{\alpha}}{(b-a)^{\alpha}} L_{0}
$$

Case II: $\beta=1$ (this corresponds to the boundary condition $y^{\prime}(b)=L_{1}$ )

$$
y(x)-\omega^{2 \alpha}\left[I_{a^{+}}^{\alpha} I_{b^{-}}^{\alpha} y(x)-\left.\frac{(x-a)^{\alpha}}{\alpha(b-a)^{\alpha-1}} D_{a^{+}}^{1-\alpha} I_{b^{-}}^{\alpha} y(x)\right|_{x=b}\right]=\frac{(x-a)^{\alpha}}{\alpha(b-a)^{\alpha-1}} L_{1}
$$

Case III: $\beta=\alpha$

$$
y(x)-\omega^{2 \alpha} I_{a^{+}}^{\alpha} I_{b^{-}}^{\alpha} y(x)=\frac{(x-a)^{\alpha}}{\Gamma(\alpha+1)} L_{\alpha}
$$

\section{Numerical solution}

In this part we present a numerical for Eq. (27). We introduce the following grid of nodes: $a=x_{0}<x_{1}<\ldots<x_{i}<\ldots<x_{n}=b$, where $x_{i}=a+i \Delta x, i=0,1, \ldots, n$ and $\Delta x=(b-a) / n$. Additionally, we denote the value of function $y(x)$ at the node $x_{i}$ by $y_{i}=y\left(x_{i}\right)$. In every grid node $x_{i}$, Eq. (27) is replaced by the algebraic equation and then we obtain the following system of $n+1$ equations 


$$
\begin{aligned}
y\left(x_{i}\right) & -\omega^{2 \alpha}\left[\left.I_{a^{+}}^{\alpha} I_{b^{-}}^{\alpha} y(x)\right|_{x=x_{i}}-\frac{\Gamma(\alpha-\beta+1)}{\Gamma(\alpha+1)} \frac{\left(x_{i}-x_{0}\right)^{\alpha}}{\left(x_{n}-x_{0}\right)^{\alpha-\beta}}\right. \\
& \times\left\{\begin{array}{ll}
\left.I_{a^{+}}^{\alpha-\beta} I_{b^{-}}^{\alpha} y(x)\right|_{x=x_{n}} & \text { for } \alpha>\beta \\
0 & \text { for } \alpha=\beta \\
\left.D_{a^{+}}^{\beta-\alpha} I_{b^{-}}^{\alpha} y(x)\right|_{x=x_{n}} & \text { for } \alpha<\beta
\end{array}\right]=\frac{\Gamma(\alpha-\beta+1)}{\Gamma(\alpha+1)} \frac{\left(x_{i}-x_{0}\right)^{\alpha}}{\left(x_{n}-x_{0}\right)^{\alpha-\beta}} L_{\beta}
\end{aligned}
$$

Next, the numerical approximation for $\left.I_{a^{+}}^{\alpha} I_{b^{-}}^{\alpha} y(x)\right|_{x=x_{i}},\left.I_{a^{+}}^{\alpha-\beta} I_{b^{-}}^{\alpha} y(x)\right|_{x=x_{n}}$ and $\left.D_{a^{+}}^{\beta-\alpha} I_{b^{-}}^{\alpha} y(x)\right|_{x=x_{n}}$ should be properly determined. In this order we use the numerical schemes that are based on the trapezoidal rule of integration $[22,24]$. The schemes were introduced earlier in the paper [15]. For both fractional integral operators (5) and (6) for function $f(x)$ we have:

- at node $x_{0}:\left.I_{a^{+}}^{\alpha} f(x)\right|_{x=x_{0}}=0$ (it directly results from definition),

- for other nodes $x_{i}(i=1,2, \ldots, n)$, the left-side Riemann-Liouville integral (5) is approximated by the formula

$$
\begin{aligned}
& \left.I_{a^{+}}^{\alpha} f(x)\right|_{x=x_{i}}=\frac{1}{\Gamma(\alpha)} \int_{0}^{x_{i}} \frac{f(\tau)}{\left(x_{i}-\tau\right)^{1-\alpha}} d \tau=\frac{1}{\Gamma(\alpha)} \sum_{j=0}^{i-1} \int_{x_{j}}^{x_{j+1}} \frac{f(\tau)}{\left(x_{i}-\tau\right)^{1-\alpha}} d \tau \\
& \cong \frac{1}{\Gamma(\alpha)} \sum_{j=0}^{i-1} \int_{x_{j}}^{x_{j+1}} \frac{f_{j}+\left(\tau-x_{j}\right)\left(f_{j+1}-f_{j}\right) / \Delta x}{\left(x_{i}-\tau\right)^{1-\alpha}} d \tau=\sum_{j=0}^{i} u_{i, j}^{(\alpha)} f_{j}
\end{aligned}
$$

where

$$
\begin{aligned}
u_{i, j}^{(\alpha)} & =\frac{(\Delta x)^{\alpha}}{\Gamma(2+\alpha)} \\
& \times \begin{cases}0 & \text { for } i=0 \text { and } j=0 \\
i^{\alpha}(1+\alpha)+(i-1)^{1+\alpha}-i^{1+\alpha} & \text { for } i>0 \text { and } j=0 \\
(i-j+1)^{1+\alpha}-2(i-j)^{1+\alpha}+(i-j-1)^{1+\alpha} & \text { for } i>0 \text { and } 0<j<i \\
1 & \text { for } i>0 \text { and } j=i\end{cases}
\end{aligned}
$$

While, for the right-side Riemann-Liouville integral (6) we have:

- at node $x_{n}:\left.I_{b^{-}}^{\alpha} f(x)\right|_{x=x_{n}}=0$,

- for nodes $x_{i}(i=0,1, \ldots . n-1)$, the discrete values of operator (6) are calculated using the formula below 


$$
\left.I_{b^{-}}^{\alpha} f(x)\right|_{x=x_{i}} \approx \sum_{j=i}^{n} v_{i, j}^{(\alpha)} f_{j}
$$

where

$$
\begin{aligned}
& v_{i, j}^{(\alpha)}=\frac{(\Delta x)^{\alpha}}{\Gamma(2+\alpha)} \\
& \quad \times \begin{cases}0 & \text { for } i=n \text { and } j=n \\
(n-i)^{\alpha}(1+\alpha)+(n-i-1)^{1+\alpha}-(n-i)^{1+\alpha} & \text { for } i<n \text { and } j=n \\
(j-i+1)^{1+\alpha}-2(j-i)^{1+\alpha}+(j-i-1)^{1+\alpha} & \text { for } i<n \text { and } i<j<n \\
1 & \text { for } i<n \text { and } j=i\end{cases}
\end{aligned}
$$

The discrete form of the composition of both integral operators $I_{a^{+}}^{\alpha} I_{b^{-}}^{\alpha} y(x)$ at all nodes $x_{i}$ is as follows

$$
\left.I_{a^{+}}^{\alpha} I_{b^{-}}^{\alpha} y(x)\right|_{x=x_{i}} \approx \begin{cases}0 & \text { for } i=0 \\ \sum_{j=0}^{i} u_{i, j}^{(\alpha)} \sum_{k=j}^{n} v_{j, k}^{(\alpha)} y_{k}=\sum_{j=0}^{n} z_{i, j}^{(\alpha)} y_{j} & \text { for } i=1,2, \ldots, n\end{cases}
$$

where

$$
z_{i, j}^{(\alpha)}=\sum_{k=0}^{\min (i, j)} u_{i, k}^{(\alpha)} v_{k, j}^{(\alpha)}
$$

For the second operator $\left.I_{a^{+}}^{\alpha-\beta} I_{b^{-}}^{\alpha} y(x)\right|_{x=x_{n}}$, the formulas (32) and (34) can be directly applied, and thus we have

$$
\left.I_{a^{+}}^{\alpha-\beta} I_{b^{-}}^{\alpha} y(x)\right|_{x=x_{n}} \approx \sum_{j=0}^{n} u_{n, j}^{(\alpha-\beta)} \sum_{k=j}^{n} v_{j, k}^{(\alpha)} y_{k}=\sum_{j=0}^{n} z_{j}^{(\alpha, \beta)} y_{j}
$$

where

$$
z_{j}^{\prime(\alpha, \beta)}=\sum_{k=0}^{j} u_{n, k}^{(\alpha-\beta)} v_{k, j}^{(\alpha)}
$$

While, for the operator $\left.D_{a^{+}}^{\beta-\alpha} I_{b^{-}}^{\alpha} y(x)\right|_{x=x_{n}}$, we additionally need to derive the discrete form of fractional differential operator at nodes $x_{i}:\left.D_{a^{+}}^{\alpha} f(x)\right|_{x=x_{i}}$ that can be calculated as 


$$
\begin{aligned}
& \left.D_{a+}^{\alpha} f(x)\right|_{x=x_{i}}=f_{0} \frac{\left(x_{i}-x_{0}\right)^{-\alpha}}{\Gamma(1-\alpha)}+\frac{1}{\Gamma(1-\alpha)} \int_{x_{0}}^{x_{i}} \frac{f^{\prime}(\tau)}{\left(x_{i}-\tau\right)^{\alpha}} d \tau \\
& \cong f_{0} \frac{\left(x_{i}-x_{0}\right)^{-\alpha}}{\Gamma(1-\alpha)}+\frac{1}{\Gamma(1-\alpha)} \sum_{j=0}^{i-1} \frac{f_{j+1}-f_{j}}{\Delta x} \int_{x_{j}}^{x_{j+1}} \frac{1}{\left(x_{i}-\tau\right)^{\alpha}} d \tau=\sum_{j=0}^{i} w_{i, j}^{(\alpha)} f_{j}
\end{aligned}
$$

where

$$
\begin{aligned}
& w_{i, j}^{(\alpha)}=\frac{(\Delta x)^{-\alpha}}{\Gamma(2-\alpha)} \\
& \quad \times \begin{cases}0 & \text { for } i=0 \text { and } j=0 \\
(1-\alpha) i^{-\alpha}+(i-1)^{1-\alpha}-i^{1-\alpha} & \text { for } i>0 \text { and } j=0 \\
(i-j+1)^{1-\alpha}-2(i-j)^{1-\alpha}+(i-j-1)^{1-\alpha} & \text { for } i>0 \text { and } 0<j<i \\
1 & \text { for } i>0 \text { and } j=i\end{cases}
\end{aligned}
$$

and thus we have

$$
\left.D_{a^{+}}^{\beta-\alpha} I_{b^{-}}^{\alpha} y(x)\right|_{x=x_{n}} \approx \sum_{j=0}^{n} w_{n, j}^{(\beta-\alpha)} \sum_{k=j}^{n} v_{j, k}^{(\alpha)} y_{k}=\sum_{j=0}^{n} z_{j}^{\prime \prime(\alpha, \beta)} y_{j}
$$

where

$$
z_{j}^{\prime \prime(\alpha, \beta)}=\sum_{k=0}^{j} w_{n, k}^{(\beta-\alpha)} v_{k, j}^{(\alpha)}
$$

If we put the discrete schemas (36), (38) and (42) into (31) we then obtain

$$
\begin{aligned}
& y_{i}-\omega^{2 \alpha}\left[\begin{array}{ll}
0 & \text { for } i=0 \\
\sum_{j=0}^{n} z_{i, j}^{(\alpha)} y_{j} & \text { for } i=1,2, \ldots, n
\end{array}-\frac{\Gamma(\alpha-\beta+1)}{\Gamma(\alpha+1)} \frac{(i \Delta x)^{\alpha}}{(n \Delta x)^{\alpha-\beta}}\right. \\
& \times\left\{\begin{array}{ll}
\sum_{j=0}^{n} z_{j}^{\prime(\alpha, \beta)} y_{j} & \text { for } \alpha>\beta \\
0 & \text { for } \alpha=\beta \\
\sum_{j=0}^{n} z_{j}^{\prime \prime(\alpha, \beta)} y_{j} & \text { for } \alpha<\beta
\end{array}\right]=\frac{\Gamma(\alpha-\beta+1)}{\Gamma(\alpha+1)} \frac{(i \Delta x)^{\alpha}}{(n \Delta x)^{\alpha-\beta}} L_{\beta}
\end{aligned}
$$

for $i=0,1, \ldots, n$. 
The above system of equations (44) can be written in the matrix form

$$
\mathbf{A} \cdot \mathbf{y}=\mathbf{b}
$$

where $\mathbf{y}=\left[y_{0}, y_{1}, \ldots, y_{n}\right]^{\mathrm{T}}$ and the coefficients in matrices $\mathbf{A}$ and $\mathbf{b}$ are the following

$A_{i, j}=\delta_{i, j}-\omega^{2 \alpha}\left[\left\{\begin{array}{ll}0 & \text { for } i=0 \\ z_{i, j}^{(\alpha)} & \text { for } i=1,2, \ldots, n\end{array}-\frac{\Gamma(\alpha-\beta+1)}{\Gamma(\alpha+1)} \frac{(i \Delta x)^{\alpha}}{(n \Delta x)^{\alpha-\beta}}\left\{\begin{array}{ll}z_{j}^{\prime(\alpha, \beta)} & \text { for } \alpha>\beta \\ 0 & \text { for } \alpha=\beta \\ z_{j}^{\prime \prime(\alpha, \beta)} & \text { for } \alpha<\beta\end{array}\right]\right.\right.$

$b_{i}=\frac{\Gamma(\alpha-\beta+1)}{\Gamma(\alpha+1)} \frac{(i \Delta x)^{\alpha}}{(n \Delta x)^{\alpha-\beta}} L_{\beta}$

for $i=0,1, \ldots, n, j=0,1, \ldots, n$ and

$$
\delta_{i, j}= \begin{cases}1 & \text { for } i=j \\ 0 & \text { for } i \neq j\end{cases}
$$

\section{Results}

In this section we present the results of example computations obtained by our numerical scheme to the considered equation (27). We present several examples of calculations for different values of parameters $\alpha, \beta, \omega$. In all examples we assumed $a=0, b=1$ and $L_{\beta}=1$. The domain $x \in[0,1]$ has been divided into $n=1000$ subintervals. The values of the parameters used in Eq. (27) are given in the plot legends.

The simulation results as the plots of function $y(x)$ are given below. In Figures 1-4 we present results of the numerical solution of Eq. (27) for the fixed parameter $\omega \in\{0,1,5,15\}$ in the successive Figures, various values of order $\alpha$ and three values of parameter $\beta \in\{0,1, \alpha\}$ representing three particular cases (28), (29) and (30). While in Figure 5, the solutions of this equation for $\alpha=1, \omega \in\{0,1,10,20\}$, and $\beta \in\{0,0.2,0.4,0.6,0.8,1\}$ are given.

\section{Conclusions}

In this paper the fractional oscillator equation (in which the left and right fractional derivatives of order $\alpha \in(1 / 2,1]$ occur simultaneously) has been considered. This equation supplemented by adequate boundary conditions (the Dirichlet boundary condition on the left side of the considered 1D domain and various types of boundary conditions on the right side) was transformed into the integral form. 

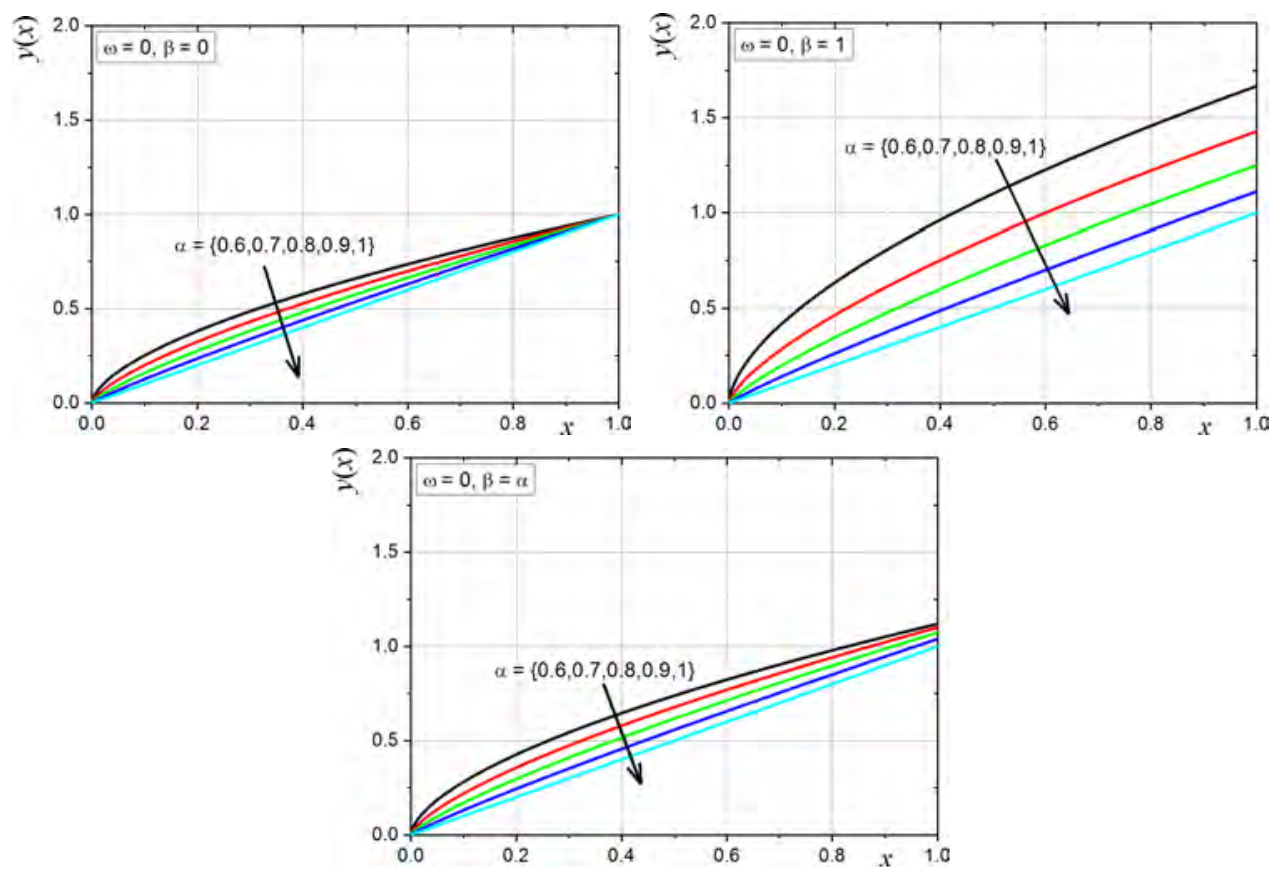

Fig. 1. Numerical solution of Eq. (27) for $\omega=0, \alpha \in\{0.6,0.7,0.8,0.9,1\}, \beta \in\{0,1, \alpha\}$, $a=0, b=1$ and $L_{\beta}=1$
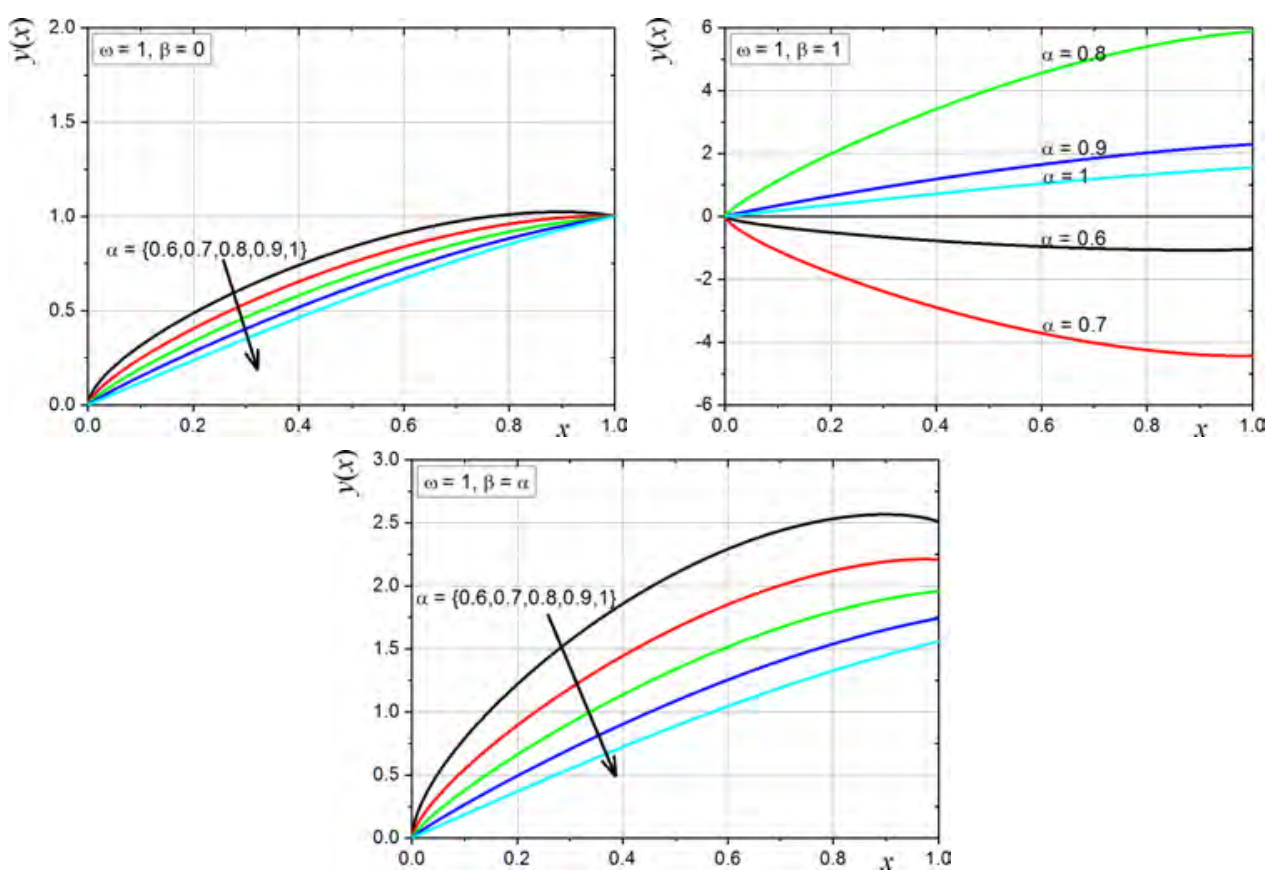

Fig. 2. Numerical solution of Eq. (27) for $\omega=1, \alpha \in\{0.6,0.7,0.8,0.9,1\}, \beta \in\{0,1, \alpha\}$, $a=0, b=1$ and $L_{\beta}=1$ 


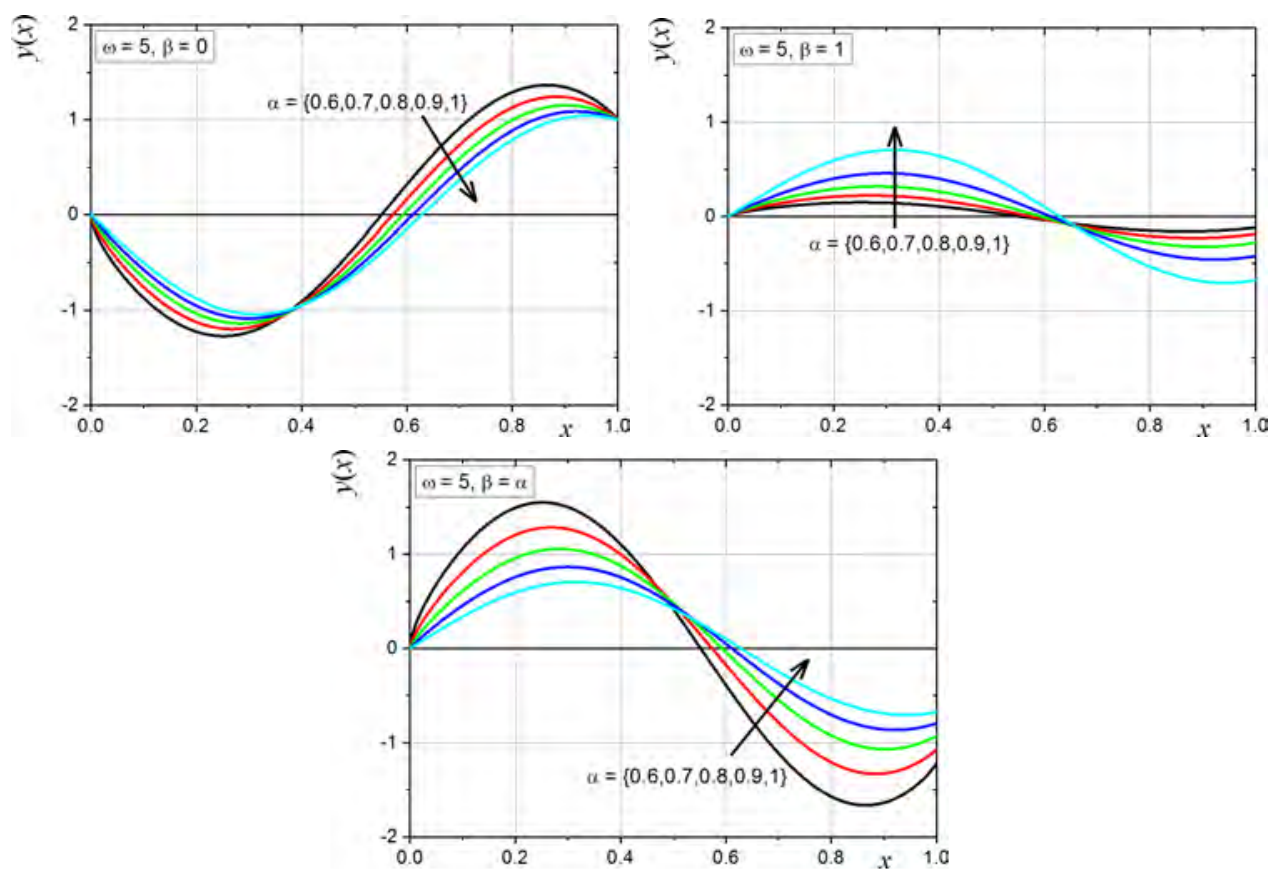

Fig. 3. Numerical solution of Eq. (27) for $\omega=5, \alpha \in\{0.6,0.7,0.8,0.9,1\}, \beta \in\{0,1, \alpha\}$, $a=0, b=1$ and $L_{\beta}=1$
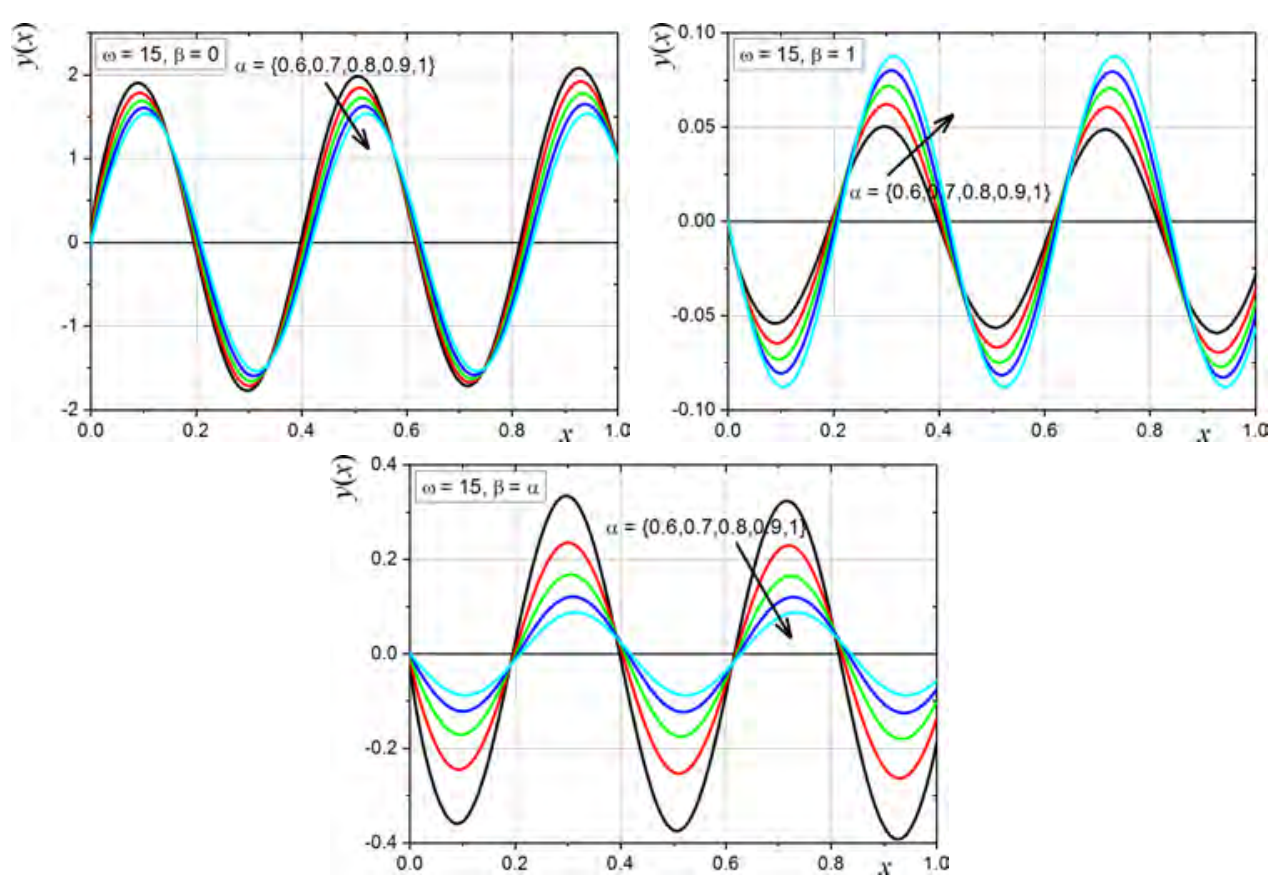

Fig. 4. Numerical solution of Eq. (27) for $\omega=15, \alpha \in\{0.6,0.7,0.8,0.9,1\}, \beta \in\{0,1, \alpha\}$, $a=0, b=1$ and $L_{\beta}=1$ 

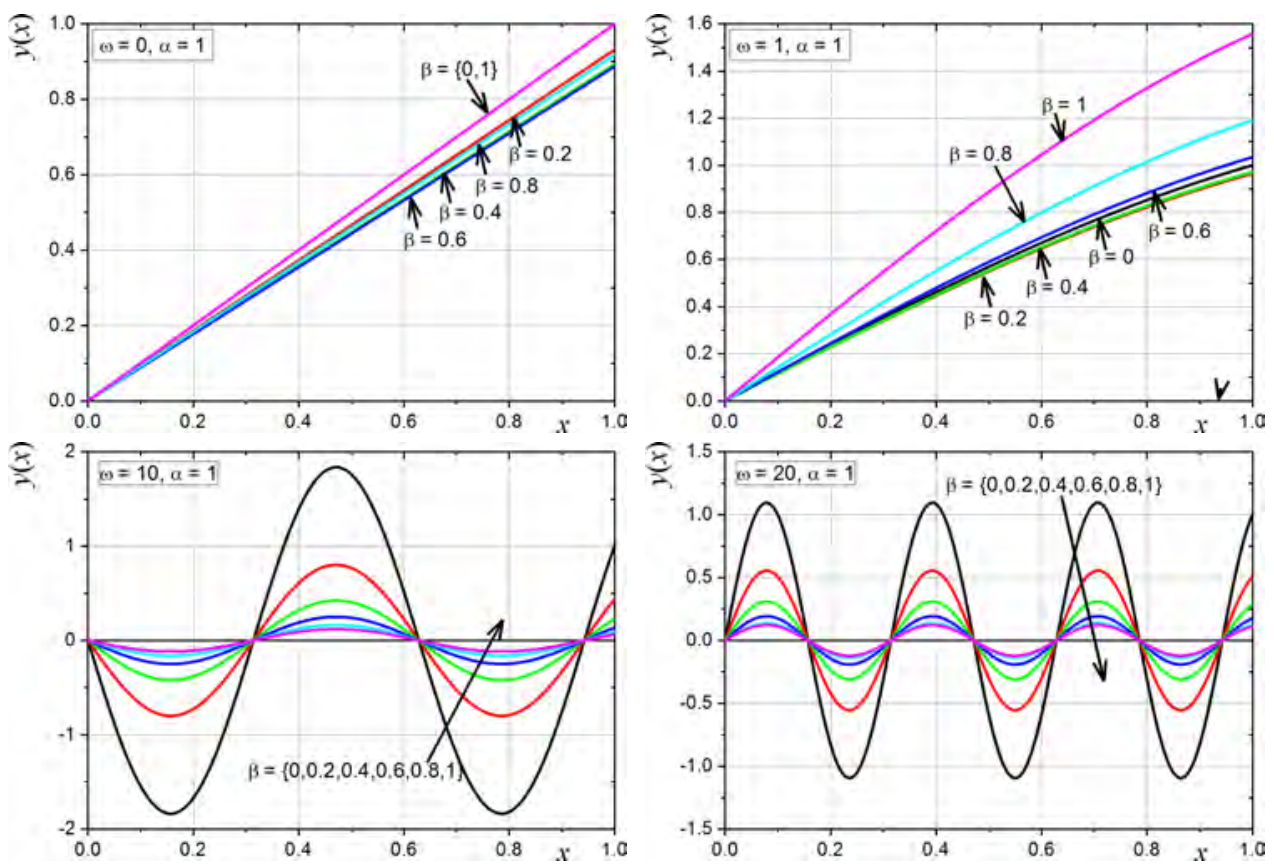

Fig. 5. Numerical solution of Eq. (27) for $\alpha=1, \omega \in\{0,1,10,20\}$, $\beta \in\{0,0.2,0.4,0.6,0.8,1\}, a=0, b=1$ and $L_{\beta}=1$

Three particular cases of order of the fractional derivative, that occur in the boundary condition, deserved particular attention. The solution of the derived integral equation was determined in a numerical way.

One of the aims of our work was to analyse the impact of different types of boundary conditions (the Dirichlet, Neumann and fractional one) on the solution of the considered boundary value problem. In the cases when the analytical solutions are known and easily determinable (i.e. $\alpha=1$ and $\beta=0$ or $\beta=1$ ), the numerical solutions are in good agreement with them. Also, for $\alpha=1$ and fractional order of $\beta$ (the known analytical solution can be numerically approximated), the numerical solutions of the integral equation seem to be correct.

We hope that the presented fractional boundary value problem in this work allows for the modelling of the oscillations in any complex systems where the classical oscillator models fail. The numerical solution of this problem can be helpful in the analysis of the behaviour of this solution depending on various values of the fractional orders $\alpha$ and $\beta$, different values of $\omega$ and parameters in the boundary conditions.

\section{References}

[1] Klimek M., On Solutions of Linear Fractional Differential Equations of a Variational Type, The Publishing Office of the Czestochowa University of Technology, Czestochowa 2009.

[2] Malinowska A.B., Torres D.F.M., Introduction to the Fractional Calculus of Variations, Imperial College Press, London 2012. 
[3] Malinowska A.B., Odzijewicz T., Torres D.F.M., Advanced Methods in the Fractional Calculus of Variations, Springer International Publishing, London 2015.

[4] Agrawal O.P., Formulation of Euler-Lagrange equations for fractional variational problems, J. Math. Anal. Appl. 2002, 272, 368-379.

[5] Baleanu D., Trujillo J.J., On exact solutions of a class of fractional Euler-Lagrange equations, Nonlinear Dyn. 2008, 52, 331-335.

[6] Bourdin L., Existence of a weak solution for fractional Euler-Lagrange equations, J. Math. Anal. Appl. 2013, 399, 239-251.

[7] Kamocki, R. Majewski, M., On the existence and continuous dependence on parameter of solutions to some fractional dirichlet problem with application to lagrange optimal control problem, J. Optim. Theory Appl. 2017, 174, 32-46.

[8] Klimek M., Malinowska A. B., Odzijewicz T., Applications of the fractional Sturm-Liouville problem to the space-time fractional diffusion in a finite domain, Fract. Calc. Appl. Anal. 2016, 19(2), 516-550.

[9] Riewe F., Nonconservative Lagrangian and Hamiltonian mechanics, Phys. Rev. E 1996, 53, 1890-1899.

[10] Ciesielski M., Klimek M., Blaszczyk T., The Fractional Sturm-Liouville problem - numerical approximation and application in fractional diffusion, J. Comput. Appl. Mathem. 2017, 317, 573-588.

[11] Fazli H., Bahrami F., On the steady solutions of fractional reaction-diffusion equations, Filomat 2017, 31(6), 1655-1664.

[12] Sumelka W., Blaszczyk T., Fractional continua for linear elasticity, Arch. Mech. 2014, 66(3), 147-172.

[13] Sumelka W., Fractional calculus for continuum mechanics - anisotropic non-locality, Bulletin of the Polish Academy of Sciences Technical Sciences 2016, 64(2), 361-372.

[14] Baleanu D., Asad J.H., Petras I., Fractional Bateman-Feshbach Tikochinsky oscillator, Commun. Theor. Phys. 2014, 61, 221-225.

[15] Blaszczyk T., Ciesielski M., Fractional oscillator equation - transformation into integral equation and numerical solution, Appl. Mathem. Comput. 2015, 257, 428-435.

[16] Agrawal O.P., Hasan M.M., Tangpong X.W., A numerical scheme for a class of parametric problem of fractional variational calculus, J. Comput. Nonlinear Dyn. 2012, 7, 021005-1-021005-6.

[17] Baleanu D., Diethelm K., Scalas E., Trujillo J.J., Fractional Calculus Models and Numerical Methods, World Scientific, Singapore 2012.

[18] Blaszczyk T., Ciesielski M., Numerical solution of fractional Sturm-Liouville equation in integral form, Fract. Calc. Appl. Anal. 2014, 17(2), 307-320.

[19] Bourdin L., Cresson J., Greff I., Inizan P., Variational integrator for fractional Euler-Lagrange equations, Appl. Numer. Mathem. 2013, 71, 14-23.

[20] Xu Y., Agrawal O.P., Models and numerical solutions of generalized oscillator equations, J. Vib. Acoust. 2014, 136(5) Article ID 050903.

[21] Kilbas A.A., Srivastava H.M., Trujillo J.J., Theory and Applications of Fractional Differential Equations, Elsevier, Amsterdam 2006.

[22] Oldham K.B., Spanier J., The Fractional Calculus: Theory and Applications of Differentiation and Integration to Arbitrary Order, Academic Press, San Diego 1974.

[23] Podlubny I., Fractional Differential Equations, Academic Press, San Diego 1999.

[24] Press W.H., Teukolsky S.A., Vetterling W.T., Flannery B.P., Numerical Recipes: The Art of Scientific Computing (3rd ed.), Cambridge University Press, New York 2007. 